\title{
Action video games and improved attentional control: Disentangling selection- and response-based processes
}

\author{
Joseph D. Chisholm • Alan Kingstone
}

Published online: 14 March 2015

(C) Psychonomic Society, Inc. 2015

\begin{abstract}
Research has demonstrated that experience with action video games is associated with improvements in a host of cognitive tasks. Evidence from paradigms that assess aspects of attention has suggested that action video game players (AVGPs) possess greater control over the allocation of attentional resources than do non-video-game players (NVGPs). Using a compound search task that teased apart selectionand response-based processes (Duncan, 1985), we required participants to perform an oculomotor capture task in which they made saccades to a uniquely colored target (selectionbased process) and then produced a manual directional response based on information within the target (response-based process). We replicated the finding that AVGPs are less susceptible to attentional distraction and, critically, revealed that AVGPs outperform NVGPs on both selection-based and response-based processes. These results not only are consistent with the improved-attentional-control account of AVGP benefits, but they suggest that the benefit of action video game playing extends across the full breadth of attention-mediated stimulus-response processes that impact human performance.
\end{abstract}

Keywords Eye movements · Visual attention · Attentional capture $\cdot$ Visual search $\cdot$ Cognitive and attentional control

Over the past decade, researchers have taken an interest in the impact that action video game experience has on cognition. Since action video games are typically fast-paced, require

J. D. Chisholm · A. Kingstone

Department of Psychology, University of British Columbia,

Vancouver, British Columbia, Canada

J. D. Chisholm $(\square)$

University of British Columbia, 2136 West Mall, Vancouver, British

Columbia V6T 1Z4, Canada

e-mail: jchisholm@psych.ubc.ca players to accurately select relevant information and make split-second decisions in contexts that are visually complex, and are attentionally demanding, these games have been targeted as an ideal candidate to assess the effect of experience on cognition. Experience with action video games has been linked to a variety of visual and cognitive benefits, with much of this work emphasizing improvements in tasks that engage selective-attention processes (for reviews, see Bavelier, Green, Pouget, \& Schrater, 2012; Hubert-Wallander, Green, \& Bavelier 2011a; Spence \& Feng, 2010). For example, relative to non-video-game players (NVGPs), action video game players (AVGPs) have demonstrated differences in the spatial distribution of attention (Dye \& Bavelier, 2010; Feng, Spence, \& Pratt, 2007; Green \& Bavelier, 2003, 2006a; Greenfield, DeWinstanley, Kilpatrick, \& Kaye, 1994), as well as improvements in visual search performance and distractor inhibition (Cain, Prinzmetal, Shimamura, \& Landau, 2014; Castel, Pratt, \& Drummond, 2005; Chisholm, Hickey, Theeuwes, \& Kingstone, 2010; Chisholm \& Kingstone, 2012; Green \& Bavelier, 2007; Hubert-Wallander, Green, Sugarman, \& Bavelier 2011b; Krishnan, Kang, Sperling, \& Srinivasan, 2013; Mishra, Zinni, Bavelier, \& Hillyard, 2011). A number of these effects have also been demonstrated in NVGPs following training with action video games, providing evidence that a causal relationship exists between action video game experience and improved performance (e.g., Feng et al. 2007; Green \& Bavelier, 2003, 2006a, 2006b, 2007; Li, Polat, Makous, \& Bavelier, 2009; however, see Boot, Blakely, \& Simons, 2011, and Kristjánsson, 2013, for criticisms, and Boot, Kramer, Simons, Fabiani, \& Gratton, 2008, for failure to train).

Although researchers in the field continues to investigate the various conditions under which AVGPs outperform NVGPs, a greater emphasis has been placed on furthering our understanding of the mechanisms underlying the improvements demonstrated by AVGPs. A prominent account for the performance differences observed between AVGPs and 
NVGPs attributes them to improvements in the controlled allocation of attentional resources (Hubert-Wallander, Green, \& Bavelier 2011a; however, see Bavelier et al. 2012, and Green \& Bavelier, 2012, for a discussion of a recently proposed learning-to-learn account). Evidence in support of this account has largely emerged from performance differences in tasks that require participants to covertly engage visuospatial attention (i.e., in which eye movements are restricted). Although informative, such paradigms typically require one to infer attentional effects on the basis of less direct measures of attention (e.g., manual response times [RTs] and accuracy measures). Therefore, although evidence acquired from covert attentional paradigms has provided support for an attentionbased account of AVGP benefits, the details as to how improved control is manifested could often be clarified.

Take, as an example, our recent report that AVGPs were better able to resist distraction from a task-irrelevant singleton (Chisholm et al. 2010). This finding was consistent with an attention-based account of AVGPs' performance improvements, but due to the covert nature of the task, we were unable to determine how that improved performance was being realized. One possibility, which we favored, was that AVGPs and NVGPs were both being captured by the irrelevant distractor in equivalent bottom-up manners (e.g., Theeuwes, 1991, 1992, 2004), but that AVGPs were more effective at disengaging from the distractor and reorienting attention to the target. An alternative explanation was that AVGPs were better able to exert top-down control over their attention in order to avoid being distracted by the irrelevant singleton. In a follow-up study (Chisholm \& Kingstone, 2012), we employed an oculomotor capture paradigm to discriminate which of these two scenarios was correct. Contrary to our initial hypothesis, we found that AVGPs were better able to avoid capture by the irrelevant singleton, and when they were captured, they were no more effective at disengaging from the distractor than were NVGPs. In this way, one can use an overt measure of attention to better understand how the differences between AVGPs and NVGPs are realized within a covert attention paradigm.

Motivated by the goal to better understand the specifics of how improved attentional control is realized by AVGPs, in the present investigation we compared AVGP and NVGP performance in a modified version of the oculomotor capture task used by Chisholm and Kingstone (2012). That task was what Duncan (1985) referred to as a simple search task. In simple search, the stimulus information that separates a target from distractors is sufficient to determine the correct final response. In Chisholm and Kingstone (2012), deciding which of the items was colored blue was sufficient to execute a correct eye movement to the target. This can be contrasted with compound search, in which the stimulus information separating a target from distractors does not identify which of the possible responses to choose. In other words, the defining attribute of the target (what distinguishes it from the distractors) is different from the reported attribute (the attribute described in the final response). Note that this distinction between simple and compound search closely resembles Broadbent's (1971) distinction between the stimulus set and response set. In the present article, we use target selection to refer to the selection of a target based on its defining attribute, and target response to refer to the decision of the final correct response. It is important to note that the response measure was not meant to reflect participants' ability to simply execute a motor action. Previous work has provided evidence that AVGP and NVGP differences are not fully explained by such an account (Green, Pouget, \& Bavelier, 2010; Hubert-Wallander, Green, Sugarman, \& Bavelier 2011b). Instead, the response measure was based on the time taken to make a discriminatory response decision, which can be influenced by the commitment of attention (e.g., Allport, 1993; Duncan, 1996; Kahneman, 1973; Posner \& Rothbart, 2007).

Thus, although Chisholm and Kingstone (2012) discovered that AVGPs outperform NVGPs by avoiding capture by the distractor, because they conducted a simple search task, thereby confounding a target's defining and reported attributes, it remained unclear whether AVGPs' performance benefit arises during the selection of the target (its defining attribute) or the decision of the final response (its reported attribute). To tease these two processes apart, we modified Chisholm and Kingstone's (2012) simple search task to make it a compound search task. Specifically, participants searched for a target defined by its color (measured by eye movement performance) and then identified the location of an indent within the target (measured by a two-alternative forced choice button-press response). This compound search allowed us to separate and measure the two operations, target selection and response, in AVGPs and NVGPs. By comparing AVGP and NVGP performance on these two measures, we could determine whether AVGP visual search performance is associated with improvements in target selection (selection of the target's color), target response (report of the target's indent), or both. If only the selection of a target's defining attribute were improved, we predicted that we would observe more efficient saccades to target stimuli with equivalent manual RTs once the target was reached. The reverse would be predicted if the decision time of the reported attribute were facilitated for AVGPs. If video game playing benefits both target selection and response processes, then enhanced eye and manual RTs would be observed for the AVGPs as compared to the NVGPs.

\section{Method}

Participants

The data from 57 undergraduate male participants (1730 years old, mean $=20.5)$, recruited from the University of 
British Columbia, are reported. Recruitment largely involved explicitly asking for AVGPs and NVGPs to participate, and participants completed a questionnaire to assess their video game experience prior to completing the task. Those who reported playing a minimum of $3 \mathrm{~h}$ per week of action video games over the last six months were defined as AVGPs. NVGPs were defined as those who reported little to no action video game playing over the past six months. Altogether, AVGPs $(n=28)$ reported playing an average of approximately $8 \mathrm{~h}$ of action games per week (e.g., Counter-Strike, Call of Duty, or Halo). In contrast, NVGPs $(n=29)$ reported playing no action video games and an average of approximately $1 \mathrm{~h}$ of nonaction games per week. All participants provided written informed consent, reported normal or corrected-to-normal vision, and received course credit or monetary compensation for their participation.

\section{Apparatus and stimuli}

The visual stimuli were gray and blue circles on a black background, viewed from a chinrest positioned $65 \mathrm{~cm}$ before a 17in. LCD monitor. An EyeLink 1000 (SR Research Ltd., Ottawa, ON, Canada) tracked and recorded eye movements at $1000 \mathrm{~Hz}$. The display consisted of six circles evenly spaced around an imaginary circle with a circumference of $14.7^{\circ}$ of visual angle. Each circle $\left(2.35^{\circ}\right)$ contained an inner black square $\left(0.3^{\circ}\right)$. On half of the trials, an additional blue circle, identical to the other nontarget items in the display, abruptly appeared on the imaginary circle at an angle of $90^{\circ}$ or $150^{\circ}$ from the target. Once participants had initiated an eye movement, a small triangular indent (four pixels) was made on either the right or the left side of the black square at the center of the target circle. Pilot testing indicated that the indent was only visible if fixated. Participants used a standard computer mouse to input manual responses.

\section{Procedure}

Prior to beginning the task, participants were instructed that each display would consist of one target (gray circle) among five nontargets (blue circles) and that they were required to make an eye movement to the location of the target circle. Following target selection (i.e., once fixating the target), participants were required to provide a manual response, indicating whether an indent was present on the left or the right side of the square within the target. Participants used the left and right mouse buttons to indicate whether the indent was on the left or the right side of the square, respectively. Participants were not informed that an abrupt-onset distractor could appear, but they were encouraged to respond as quickly and accurately as possible, in terms of both their eye movements and manual responses.
Each trial began with a central fixation point $\left(0.7^{\circ}\right)$ presented for $150 \mathrm{~ms}$, followed by the appearance of six gray circles. After 2,500 ms, all but one gray circle changed to blue. The target appeared at each of the possible six positions equally often. On half of the trials, an additional blue circle (abrupt onset) was added to the display at the time that the other circles turned blue. The abrupt onset appeared equal numbers of times either $90^{\circ}$ or $150^{\circ}$ from the target. After the participant made a manual response (or after 2,000 ms, whichever came first), the screen went blank for $500 \mathrm{~ms}$, signaling the trial's end (Fig. 1).

Participants completed a practice session of 12 trials and were then questioned to confirm that they could identify the target amongst the nontargets and that they could discriminate the location of the indent within the target. Participants then completed four blocks of 48 trials (192 total test trials). Before each block, a nine-point eye calibration was performed. To assess participants' ability to correctly select the defining attribute of a target, initial saccades that landed within a $70^{\circ}$ window centered on the target (i.e., $35^{\circ}$ on either side) were recorded as correct saccades. Initial saccades landing within $70^{\circ}$ of the onset were recorded as capture trials. Other eye movements (excluding blinks) were recorded as errors. At the end of each block, participants were presented with their average manual RT for that block. Participants were asked to read these times to the experimenter in order to encourage them to remain motivated to respond quickly and accurately.

\section{Results}

The following trials were excluded from analysis: Trials on which participants initiated a saccade sooner than $100 \mathrm{~ms}$ or later than $500 \mathrm{~ms}$, trials on which participants failed to maintain initial fixation within $2^{\circ}$ of the central fixation prior to target presentation, and trials with initial saccade amplitudes less than $2^{\circ}$ or saccade velocities slower than $30^{\circ} / \mathrm{s}$. This resulted in the loss of $13.7 \%$ of the trials $(15.1 \%$ from AVGPs and $12.5 \%$ from NVGPs, $p>.05$ ).

\section{Selection analysis}

To assess whether AVGPs and NVGPs differed in their abilities to efficiently select a relevant feature, we conducted a $2 \times 2$ repeated measures analysis of variance (ANOVA) on saccade accuracies, with Onset Presence (present vs. absent) and Video Game Experience (AVGP vs. NVGP) as factors. This analysis of saccade accuracies revealed a main effect of onset presence $[F(1,55)=300.70, p<.001]$ and a marginal effect of video game experience $[F(1,55)=3.64, p<.07]$. Importantly, a significant interaction was observed $[F(1,55)=5.72, p<.05]$; whereas AVGPs and NVGPs demonstrated comparable accuracies when no distractor appeared in the display, NVGPs 
Fig. 1 Sequence of events and examples of search displays for onset-absent and -present trials. Black circles appeared as blue in the actual displays

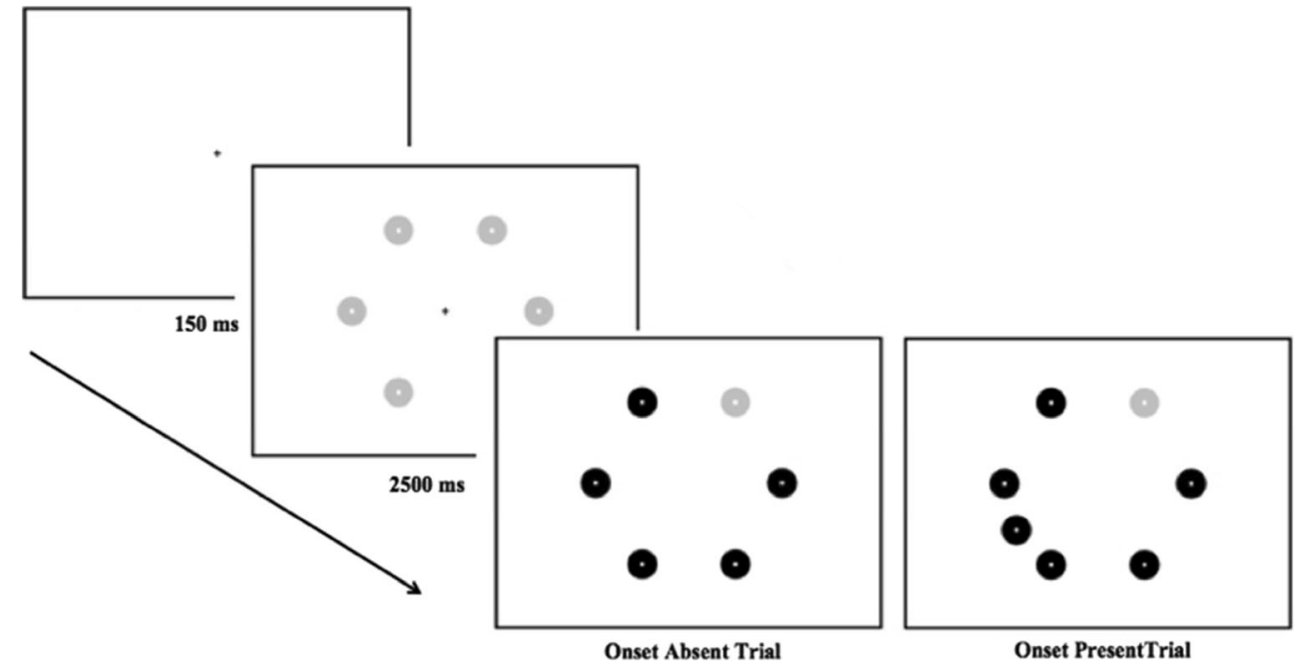

demonstrated a greater detriment in saccade accuracy than did AVGPs when a distractor was present (Fig. 2). A follow-up analysis revealed that this difference was due to a difference in the oculomotor capture experienced by both groups $[t(55)=$ 2.34, $p<.05]$ : AVGPs produced fewer incorrect saccades toward the abrupt onset (37.7\%) than did NVGPs (47.5\%). ${ }^{1}$

To further probe for differences in selection efficiency between the groups, another $2 \times 2$ repeated measures ANOVA was conducted on saccade latencies, with Onset Presence (present vs. absent) and Video Game Experience (AVGP vs. NVGP) as factors. For this analysis, only the trials on which participants were not captured by the abrupt onset were included in the distractor-present variable. Saccade latencies when captured did not differ between the groups $(p>.05)$; however, because latencies were significantly shorter for captured than for noncaptured saccades ( 219 vs. $242 \mathrm{~ms}$, respectively; $p<.05)$, this created an imbalance when comparing latencies across groups that differed in the amounts of capture experienced. The results revealed no main effect of onset presence $[F(1,55)=2.03, p>.05]$, a marginal main effect of video game experience $[F(1,55)=3.01, p<.09]$, and no significant interaction $[F(1,55)<1$; see Table 1]. These results indicate that AVGPs and NVGPs differed only marginally in the times taken to initiate a saccade, despite the differences observed in saccade accuracy. Thus, the results are not accounted for by a

\footnotetext{
$\overline{{ }^{1} \text { An additional }} 2 \times 2$ analysis was conducted on the oculomotor capture data with the factors Video Game Experience (AVGP vs. NVGP) and Onset Distance (90 vs. $150 \mathrm{deg}$ ). This analysis again revealed less overall capture in AVGPs than in NVGPs $[F(1,55)=5.24, p<.05]$, and less overall capture when the onset appeared $90 \mathrm{deg}$ rather than $150 \mathrm{deg}$ from the target $[F(1,55)=17.16, p<.001]$; however, the two factors did not interact $[F(1,55)=1.70, p>.05]$. Also, although this was not critical to the primary selection-versus-response question, an analysis was conducted of the times needed to correct a captured saccade. Replicating previous work (Chisholm \& Kingstone, 2012), AVGPs and NVGPs did not differ in the times that they needed to correct a captured saccade $(80.1$ vs. $88.5 \mathrm{~ms}$, respectively; $p>.05$ ).
}

speed-accuracy trade-off. If anything, AVGPs' saccades were both faster and more accurate.

\section{Response analysis}

Trials on which participants made saccade errors (i.e., anywhere other than toward either the target or the abrupt onset) were not included in the analysis of manual RTs. In addition, trials were excluded from any analyses if participants made an incorrect manual response or if RTs were 2.5 standard deviations from the within-subjects means (loss of $5.0 \%$ of the trials). To acquire a measure of response selection without contamination from all of the stages that preceded the response, responses were standardized to the time of arrival at the target. Thus, the manual $R T$ refers to the time taken to respond to the location of the indent from the moment the target was fixated.

To compare response selection efficiencies across AVGPs and NVGPs, $2 \times 2$ repeated measures ANOVAs were conducted on both manual RTs and manual response errors, with

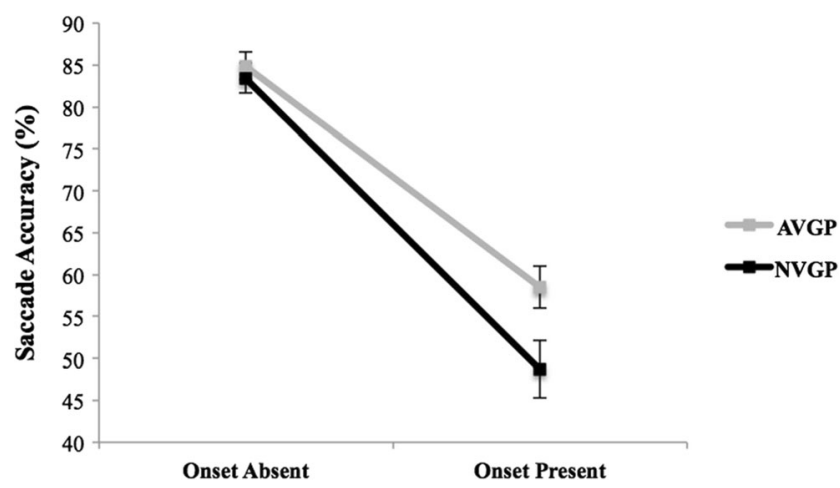

Fig. 2 Average saccade accuracy for trials on which an abrupt onset was either absent or present. Action video game players (AVGPs) produced more-accurate saccades than did non-video-game players (NVGPs) when an abrupt onset was present in the display $(p<.05)$. Error bars represent the standard errors of the means 
Table 1 Summary of action video game player (AVGP) and nonvideo-game player (NVGP) task performance (standard errors of the means in parentheses)

\begin{tabular}{|c|c|c|c|c|c|}
\hline \multirow[b]{2}{*}{ Group } & \multirow{2}{*}{$\begin{array}{l}\text { Oculomotor } \\
\text { Capture }\end{array}$} & \multicolumn{2}{|c|}{ Saccade Latency (ms) } & \multicolumn{2}{|c|}{ Manual RT (ms) } \\
\hline & & $\begin{array}{l}\text { Onset } \\
\text { Absent }\end{array}$ & $\begin{array}{l}\text { Onset } \\
\text { Present }\end{array}$ & $\begin{array}{l}\text { Onset } \\
\text { Absent }\end{array}$ & $\begin{array}{l}\text { Onset } \\
\text { Present }\end{array}$ \\
\hline AVGP & $37.7 \%(2.3)$ & $236(4.2)$ & $235(4.9)$ & $364(6.5)$ & $375(6.0)$ \\
\hline NVGP & $47.5 \%(3.5)$ & $251(6.4)$ & $248(6.5)$ & $409(10.5)$ & $415(11.4)$ \\
\hline
\end{tabular}

Onset Presence (present vs. absent) and Video Game Experience (AVGP vs. NVGP) as factors. Analysis of the manual RTs revealed main effects of distractor presence $[F(1,55)=$ $5.84, p<.05]$ and video game experience $[F(1,55)=12.79$, $p<.01]$, but no significant interaction $[F(1,55)=1.60$, $p>.05$; Table 1]. The analysis of errors revealed no main effect of distractor presence $[F(1,55)=2.64, p>.05]$ or video game experience $[F(1,55)=1.62, p>.05]$, and no significant interaction $[F(1,55)<1]$. Therefore, AVGPs and NVGPs made errors on the same percentages of trials $(3.4 \%$ vs. $2.7 \%$, respectively). These results indicate that AVGPs produced, overall, faster manual responses than NVGPs, without any significant additional cost to accuracy. ${ }^{2}$

\section{Discussion}

In the present investigation, we aimed to further understand how AVGPs outperform NVGPs in a visual search task involving distraction. Critically, we employed a task that allowed us to dissociate the behavior associated with selection- and response-based processes. To the best of our knowledge, this is the first investigation to have distinguished between these two components of visual search when comparing AVGP and NVGP performance. Our results demonstrate that AVGPs are more efficient in selecting a target - as indexed by marginally faster saccade latencies and, critically, significantly less oculomotor capture. The latter result replicates the primary finding of our previous work (Chisholm \& Kingstone, 2012) and is consistent with the notion of greater saccade control in AVGPs (West, Al-Aidroos, \& Pratt, 2013), providing evidence for the reliability of improved oculomotor control in AVGPs. In addition, the results indicate that AVGPs are also better at making quick and accurate manual responses - indexed by quicker button presses - once the target has been fixated. Thus, collectively, our results demonstrate that action video game experience is associated with improvements in both selection-based and response-based processes.

\footnotetext{
${ }^{2}$ A series of correlational analyses were also conducted on all reported performance measures with the numbers of hours that AVGPs reported playing action video games per week. No significant relationships were observed (all $r \mathrm{~s}<.11$, all $p \mathrm{~s}>.05$ ).
}

Since the likelihood of producing a reflexive saccade is associated with the availability of cognitive resources and the integrity of prefrontal cortical regions, often associated with attentional processing (e.g., Gaymard, Ploner, Rivaud, Vermersch, \& Pierrot-Deseilligny, 1998; Mitchell, Macrae, \& Gilchrist, 2002; Olk, Chang, Kingstone, \& Ro 2006; Roberts, Hager, \& Heron, 1994), the fact that AVGPs are better able to resist oculomotor capture lends further support for the notion that action video game experience is associated with greater attentional control (Hubert-Wallander, Green, \& Bavelier 2011a). This notion has been further supported by evidence demonstrating AVGPs' improved resistance to distraction at a neurophysiological level (Krishnan et al. 2013; Mishra et al. 2011; Wu et al. 2012). The basis for improved response selection is, however, equivocal. Some have argued that faster manual responses may be due to improvements in the execution of motor responses (Castel et al. 2005). This makes intuitive sense, given that AVGPs are effectively trained to be fast button-pressers. One could argue that the present demonstration of faster manual responses exhibited by AVGPs supports this claim. However, recent work has provided evidence against this postdecisional motor account as an explanatory basis for a range of AVGP advantages (Dye, Green, \& Bavelier, 2009; Green et al., 2010; Hubert-Wallander, Green, Sugarman, \& Bavelier 2011b). Instead, the evidence suggests that more efficient responding could stem from AVGPs' enhanced visual acuity (Green \& Bavelier, 2007) or ability to acquire sensory information more quickly (Appelbaum, Cain, Darling, \& Mitroff, 2013; Pohl et al. 2014; Wilms, Petersen, \& Vangkilde, 2013), both of which are gated by attentional processing and can result in more efficient perceptual decision-making (Green et al. 2010). This notion of AVGPs being more efficient at processing task-relevant information may provide a general mechanism that not only accounts for the present findings but also may provide a basis for the improved attentional control proposed to account for the breadth of AVGP benefits. For example, the improved-attentional-control explanation described above to account for more efficient selection in AVGPs could be a result of AVGPs being able to process visual information more quickly. Faster processing provides a clear advantage when one is making a perceptual decision, because this could result in response facilitation or, when a display is masked, can yield responses that are based on better-quality information (e.g., faster responses are made on the basis of representations that have faded less).

Two caveats regarding the present work need to be raised and addressed. First, given the cross-sectional nature of the present investigation, it is important to note that a degree of caution should be taken when considering the causal relationship between action video game experience and the observed effects. Therefore, conducting training studies with the present paradigm will be important for establishing a causal link between action video game experience and improved oculomotor 
control. That being said, many training studies have been conducted with other paradigms and have provided compelling evidence in favor of a causal link between action video game experience and many of the performance improvements observed in cross-sectional investigations (e.g., Feng et al. 2007; Green \& Bavelier, 2003, 2006a, 2006b, 2007; Li et al. 2009; however, see Boot et al. 2008). Second, in a recent thread of criticism of the field, some has argued that the active recruitment of AVGPs could have influenced or even caused the observed effects (Boot et al. 2011; Kristjánsson, 2013). That is, if AVGPs know that they are being recruited specifically for their expertise, they may be particularly motivated to perform better on the task. Although this raises a potentially critical concern for the reliability of the benefits demonstrated by AVGPs, it is important to note that other work has demonstrated AVGP advantages even when using covert recruitment (Chisholm \& Kingstone, under revision; Clark, Fleck, \& Mitroff, 2011; Donohue, Woldorff, \& Mitroff 2010).

\section{Conclusion}

In the present investigation, we aimed to further our understanding of how AVGPs outperform NVGPs on a visual search task. Since previous work had confounded selection-based and response-based processes, in the present study we dissociated these two processes. The results revealed that action video game experience yields benefits to both target selection and response-based processes. Specifically, replicating the results of previous work, AVGPs demonstrated reduced distraction to salient task-irrelevant stimuli. In addition, they produced more rapid manual responses. These results are consistent with the proposal that AVGPs possess greater or more efficient attentional control than do NVGPs. One particularly noteworthy aspect of the present work is that it provides insight into how this improved control is achieved, by employing a direct measure of attentional allocation. Furthermore, although there is growing interest in using video games as a rehabilitative tool, the utility of such an endeavor will be based on our understanding of the specific processes affected by video game experience. In the present investigation, we provide evidence that suggests that action video games could benefit those with deficits in either selection-based or response-based processes.

\section{References}

Allport, A. (1993). Attention and control: Have we been asking the wrong questions? A critical review of twenty-five years. In D. E. Meyer \& S. Kornblum (Eds.), Attention and performance XIV: Synergies in experimental psychology, artificial intelligence, and cognitive neuroscience (pp. 183-218). Cambridge, MA: MIT Press.
Appelbaum, L. G., Cain, M. S., Darling, E. F., \& Mitroff, S. R. (2013). Action video game playing is associated with improved visual sensitivity, but not alterations in visual sensory memory. Attention, Perception, \& Psychophysics, 75, 1161-1167. doi:10.3758/ s13414-013-0472-7

Bavelier, D., Green, C. S., Pouget, A., \& Schrater, P. (2012). Brain plasticity through the life span: Learning to learn and action video games. Annual Review of Neuroscience, 35, 391-416. doi:10. 1146/annurev-neuro-060909-152832

Boot, W. R., Blakely, D. P., \& Simons, D. J. (2011). Do action video games improve perception and cognition? Frontiers in Psychology, 2, 226. doi:10.3389/fpsyg.2011.00226

Boot, W. R., Kramer, A. F., Simons, D. J., Fabiani, M., \& Gratton, G. (2008). The effects of video game playing on attention, memory, and executive control. Acta Psychologica, 129, 387-398. doi:10.1016/j. actpsy.2008.09.005

Broadbent, D. E. (1971). Decision and stress. New York, NY: Academic Press.

Cain, M. S., Prinzmetal, W., Shimamura, A. P., \& Landau, A. N. (2014). Improved control of exogenous attention in action video game players. Frontiers in Psychology, 5, 69. doi:10.3389/fpsyg.2014.00069

Castel, A. D., Pratt, J., \& Drummond, E. (2005). The effects of action video game experience on the time course of inhibition of return and the efficiency of visual search. Acta Psychologica, 119, 217-230. doi:10.1016/j.actpsy.2005.02.004

Chisholm, J. D., Hickey, C., Theeuwes, J., \& Kingstone, A. (2010). Reduced attentional capture in action video game players. Attention, Perception, \& Psychophysics, 72, 667-671. doi:10. 3758/APP.72.3.667

Chisholm, J. D., \& Kingstone, A. (2012). Improved top-down control reduces oculomotor capture: The case of action video game players. Attention, Perception, \& Psychophysics, 74, 257-262. doi:10.3758/ s13414-011-0253-0

Clark, K., Fleck, M. S., \& Mitroff, S. R. (2011). Enhanced change detection performance reveals improved strategy use in avid action video game players. Acta Psychologica, 136, 67-72. doi:10.1016/j.actpsy. 2010.10.003

Donohue, S. E., Woldorff, M. G., \& Mitroff, S. R. (2010). Video game players show more precise multisensory temporal processing abilities. Attention, Perception, \& Psychophysics, 72, 1120-1129. doi: 10.3758/APP.72.4.1120

Duncan, J. (1985). Visual search and visual attention. In M. I. Posner \& O. S. M. Marin (Eds.), Attention and performance XI (pp. 85-106). Hillsdale, NJ: Erlbaum.

Duncan, J. (1996). Cooperating brain systems in selective perception and action. In T. Inui \& J. L. McClelland (Eds.), Attention and performance XVI: Information integration in perception and communication (pp. 549-578). Cambridge, MA: MIT Press, Bradford Books.

Dye, M. W. G., \& Bavelier, D. (2010). Differential development of visual attention skills in school-age children. Vision Research, 50, 452459. doi:10.1016/j.visres.2009.10.010

Dye, M. W. G., Green, C. S., \& Bavelier, D. (2009). Increasing speed of processing with action video games. Current Directions in Psychological Science, 18, 321-326. doi:10.1111/j.1467-8721.2009. 01660.x

Feng, J., Spence, I., \& Pratt, J. (2007). Playing an action video game reduces gender differences in spatial cognition. Psychological Science, 18, 850-855. doi:10.1111/j.1467-9280.2007.01990.x

Gaymard, B., Ploner, C. J., Rivaud, S., Vermersch, A. I., \& PierrotDeseilligny, C. (1998). Cortical control of saccades. Experimental Brain Research, 123, 159-163. doi:10.1007/s002210050557

Green, C. S., \& Bavelier, D. (2003). Action video game modifies visual selective attention. Nature, 423, 534-537. doi:10.1038/nature01647

Green, C. S., \& Bavelier, D. (2006a). Effect of action video games on the spatial distribution of visuospatial attention. Journal of 
Experimental Psychology: Human Perception and Performance, 32, 1465-1478. doi:10.1037/0096-1523.32.6.1465

Green, C. S., \& Bavelier, D. (2006b). Enumeration versus multiple object tracking: the case of action video game players. Cognition, 101, 217-245. doi:10.1016/j.cognition.2005.10.004

Green, C. S., \& Bavelier, D. (2007). Action-video-game experience alters the spatial resolution of vision. Psychological Science, 18, 88-94. doi:10.1111/j.1467-9280.2007.01853.x

Green, C. S., \& Bavelier, D. (2012). Learning, attentional control, and action video games. Current Biology, 22, R197-R206. doi:10.1016/ j.cub.2012.02.012

Green, C. S., Pouget, A., \& Bavelier, D. (2010). Improved probabilistic inference as a general learning mechanism with action video games. Current Biology, 20, 1573-1579. doi:10.1016/j.cub.2010.07.040

Greenfield, P. M., DeWinstanley, P., Kilpatrick, H., \& Kaye, D. (1994). Action video games and informal education: Effects on strategies for dividing visual attention. Journal of Applied Developmental Psychology, 15, 105-123.

Hubert-Wallander, B., Green, C. S., Sugarman, M., \& Bavelier, D. (2011a). Changes in search rate but not in the dynamics of exogenous attention in action videogame players. Attention, Perception, \& Psychophysics, 73, 2399-2412. doi:10.3758/s13414-011-0194-7

Hubert-Wallander, B., Green, C. S., \& Bavelier, D. (2011b). Stretching the limits of visual attention: The case of action video games. Wiley Interdisciplinary Reviews: Cognitive Science, 2, 222-230. doi:10. 1002/wcs. 116

Kahneman, D. (1973). Attention and effort. Englewood Cliffs, NJ: Prentice Hall.

Krishnan, L., Kang, A., Sperling, G., \& Srinivasan, R. (2013). Neural strategies for selective attention distinguish fast-action video game players. Brain Topography, 26, 83-97. doi:10.1007/s10548-012-0232-3

Kristjánsson, Á. (2013). The case for causal influences of action videogame play upon vision and attention. Attention, Perception, \& Psychophysics, 75, 667-672. doi:10.3758/s13414-013-0427-z

Li, R., Polat, U., Makous, W., \& Bavelier, D. (2009). Enhancing the contrast sensitivity function through action video game training. Nature Neuroscience, 12, 549-551. doi:10.1038/nn.2296

Mishra, J., Zinni, M., Bavelier, D., \& Hillyard, S. A. (2011). Neural basis of superior performance of video-game players in an attentiondemanding task. Journal of Neuroscience, 31, 992-998. doi:10. 1523/JNEUROSCI. 4834-10.2011
Mitchell, J. P., Macrae, C. N., \& Gilchrist, I. D. (2002). Working memory and the suppression of reflexive saccades. Journal of Cognitive Neuroscience, 14, 95-103. doi:10.1162/089892902317205357

Olk, B., Chang, E., Kingstone, A., \& Ro, T. (2006). Modulation of antisaccades by transcranial magnetic stimulation of the human frontal eye field. Cerebral Cortex, 16, 76-82. doi:10.1093/cercor/ bhi085

Pohl, C., Kunde, W., Ganz, T., Conzelmann, A., Pauli, P., \& Kiesel, A. (2014). Gaming to see: Action video gaming is associated with enhanced processing of masked stimuli. Frontiers in Psychology, 5(70), 1-9. doi:10.3389/fpsyg.2014.00070

Posner, M. I., \& Rothbart, M. K. (2007). Research on attention networks as a model for the integration of psychological science. Annual Review of Psychology, 58, 1-23. doi:10.1146/annurev.psych.58. 110405.085516

Roberts, R. J., Hager, L. D., \& Heron, C. (1994). Prefrontal cognitive processes: Working memory and inhibition in the antisaccade task. Journal of Experimental Psychology: General, 123, 374-393. doi: 10.1037/0096-3445.123.4.374

Spence, I., \& Feng, J. (2010). Video games and spatial cognition. Review of General Psychology, 14, 92-104. doi:10.1037/a0019491

Theeuwes, J. (1991). Cross-dimensional perceptual selectivity. Perception \& Psychophysics, 50, 184-193. doi:10.3758/ BF03212219

Theeuwes, J. (1992). Perceptual selectivity for color and form. Perception \& Psychophysics, 51, 599-606. doi:10.3758/BF03211656

Theeuwes, J. (2004). Top-down search strategies cannot override attentional capture. Psychonomic Bulletin \& Review, 11, 65-70. doi:10. 3758/BF03206462

West, G. L., Al-Aidroos, N., \& Pratt, J. (2013). Action video game experience affects oculomotor performance. Acta Psychologica, 142, 38-42. doi:10.1016/j.actpsy.2011.08.005

Wilms, I. L., Petersen, A., \& Vangkilde, S. (2013). Intensive video gaming improves encoding speed to visual short-term memory in young male adults. Acta Psychologica, 142, 108-118. doi:10.1016/j. actpsy.2012.11.003

Wu, S., Cheng, C. K., Feng, J., D’Angelo, L., Alain, C., \& Spence, I. (2012). Playing a first-person shooter video game induces neuroplastic change. Journal of Cognitive Neuroscience, 24, 1286-1293. doi:10.1162/jocn_a_00192 